\title{
Co-solvent Effect on Spontaneous Formation of Large Nanoscale Structures in Catanionic Mixtures in the Anionic-Rich Region
}

\author{
Farhad Golmohammadi ${ }^{1}$. Masoud Amiri ${ }^{1} \cdot$ Hussein Gharibi ${ }^{2} \cdot$ Ali Yousefi $^{2}$ • \\ Meysam Safari ${ }^{3}$
}

Received: 17 December 2018 / Accepted: 18 December 2018 / Published online: 28 November 2019

(C) The Author(s) 2019

\begin{abstract}
The aggregation behavior was investigated in mixtures of sodium dodecyl sulfate (SDS) and cetyltrimethylammonium bromide (CTAB) (anionic-rich catanionic) solutions. The study was conducted in solutions of water-ethylene glycol (EG) by means of surface tension, conductometry, cyclic voltammetry, zeta potential measurements, transmission electron microscopy (TEM) and dynamic light scattering (DLS) techniques. The degree of counterion dissociation $(\alpha)$, critical micelle concentration, aggregation numbers, interfacial properties, interparticle interaction parameters, and morphology of aggregates were determined. Based on regular solution theory, the cosolvent effects between SDS and CTAB as surfactants were also analyzed for both mixed monolayers at mixed micelles $\left(\beta^{\mathrm{M}}\right)$ and the air/liquid interface $\left(\beta^{\sigma}\right)$. It was shown that the formation of large aggregates occurred in the presence of an excess of anionic surfactant. A phase transition from cylindrical micelles to spherical micelles in the anionic-rich regime was observed with an increase in the EG volume fraction. The inter particle interactions were assessed in terms of cosolvent effects on the micellar surface charge density and the cylindrical-to-spherical morphology change. Zeta potential and size of the aggregates were determined using dynamic light scattering and confirmed the models suggested for the processes taking place in each system.
\end{abstract}

Keywords Anionic-rich region $\cdot$ Zeta potential $\cdot$ Dynamic light scattering $\cdot$ Cyclic voltammetry

Electronic supplementary material The online version of this article (https://doi.org/10.1007/s1095 3-019-00935-6) contains supplementary material, which is available to authorized users.

Farhad Golmohammadi

golmohammadifarhad@gmail.com

1 Department of Chemistry, Faculty of Science, Islamic Azad University, Kermanshah Branch, Kermanshah, Iran

2 Department of Chemistry, Faculty of Science, Tarbiat Modares University, P.O. Box 14115-175, Tehran, Iran

3 Department of Chemical Engineering, Kermanshah University of Technology, Kermanshah, Iran 


$\begin{array}{ll}\text { List of Symbols } \\ \text { CMC or } C_{1} & \text { Corresponds to the formation of large micellar aggregates } \\ C_{2} & \text { Refers to phase transition from large aggregates to normal micelles } \\ \alpha & \text { Degree of counterion dissociation } \\ \Delta \alpha & \text { Difference in degree of counterion dissociation } \\ i_{\mathrm{p}} & \text { Cathodic peak current } \\ \Gamma_{\max } & \text { Surface excess concentration } \\ A_{\min } & \text { Minimum area per head group } \\ A_{\mathrm{exp}} & \text { The corrected term is } A_{\text {min }} \\ A_{\mathrm{ideal}} & \text { Ideal mixing values } \\ \lambda_{\max } & \text { Maximum absorption wavelength } \\ \beta^{\mathrm{M}} & \text { Interaction parameter for mixed micelle } \\ \beta^{\sigma} & \text { Interaction parameter for mixed monolayer formation } \\ D_{\mathrm{agg}} & \text { Diffusion coefficient in aggregates } \\ k_{\mathrm{d}} & \text { Interparticle interaction parameter }\end{array}$

\section{Introduction}

Undoubtedly, surfactants have important roles in most areas, including cosmetics, detergents, electrochemistry, floatation agents, drug delivery, etc. [1], as well as diverse industrial applications. The chemical and surface-active properties of surfactant mixtures are superior to those of the individual surfactants [2-4]; hence, for a given application, lower amounts of surfactants are required. The understanding of the interactions among surfactants in the mixtures is the key to taking advantage of such beneficial effects. Colloidal behaviors such as micellization and surface adsorption are markedly different from those in single surfactant systems. In a system of mixed surfactants with non-ideal mixing effects in their aggregates, various micellar properties exhibit synergism or cooperative interaction $[5,6]$. This cooperative interaction or synergism is usually attributed to non-ideal mixing effects in their aggregates and modeled in the literature by employing regular solution theory (RST) with a negative interaction parameter [7-9]. The electrostatic interactions (in aqueous media) between positive and negative groups, in mixtures of anionic and cationic surfactants, give these systems many unique properties.

In comparison with the amount of work done on physical and chemical properties of surfactants in water and a polar solvent, study of surfactants in polar nonaqueous solvents and their mixtures with water is a rather limited field. Changing the solvent quality provides the opportunity to study the role of the so-called solvophobic effect as opposed to the hydrophobic effect in micellization [10]. The increasing use of surfactants in applications that require water-free or water-poor media makes this type of research more interesting [11]. Ethylene glycol (EG) with many water-like characteristics, is one of the most interesting solvents. This small molecule, with the ability to form hydrogen-bonded networks, is very similar to water although it has a completely different molecular structure. EG has a fairly high dielectric constant and high cohesive energy. These studies on the relation between the micellization process and liquid structure can lead to a better understanding of this type of process [12]. In the case of mixtures of alkyltrimethylammonium bromides and alkylpyridinium bromides, the intermolecular interactions were investigated in aggregates and mixed monolayers [13]. Based on this study, it was found that in comparison with mixed monolayers at the air/solution interface, the attractive interactions in 
mixed aggregates are even weaker. In addition, by studying the effect of a cosolvent on the behavior of anionic/cationic surfactant mixtures, in the presence of an excess of cationic surfactant [14], the percentage of cosolvent variation was evaluated as an effective tool for tuning intra-aggregate interaction. The cosolvent content can induce a transition between micelle and vesicle or a change the morphology in catanionic systems. Based on these results a powerful synergism in these catanionic mixtures in both the aggregate and monolayer states was observed. In another work, the mixtures of SDS with CTAB were studied as catanionic micelles. In this study, by means of variation in the CTAB or SDS concentrations in both rich cationic or anionic solutions, the changes in viscosity and the self-diffusion coefficient were monitored and a transition from vesicle shapes (microstructures phase) to mixed micelles (nanostructures) was observed [15].

In the present work, for the first time, to our best knowledge, the cosolvent effect on phase behavior and spontaneous super-molecular structures formation in anionic/cationic mixtures with an extra amount of anionic surfactant are being reported. Here, the interaction of SDS with CTAB surfactants has been studied comprehensively using surface tension, conductometry, cyclic voltammetry, zeta potential measurements, transmission electron microscopy (TEM) and dynamic light scattering (DLS) techniques. A combination of techniques provides important information about the structure, morphology, and phase behavior in these systems.

\section{Experimental Section}

\subsection{Materials and Sample Preparation}

Sodium dodecyl sulfate (SDS, Merck, 99\% pure) and cetyltrimethylammonium bromide (CTAB, Sigma-Aldrich, 99\% pure), and ethylene glycol (EG, Merck, 99\% pure), were used to prepare stock solutions in pure water and EG-water mixtures. Different ratios of aqueous solutions of the individual surfactants (SDS/CTAB) were prepared using doubledistilled water $\left(10 \mathrm{mmol} \cdot \mathrm{L}^{-1}\right.$ of CTBA and SDS were prepared separately and then were mixed for different ratios). All samples were prepared on the day that the measurements were carried out (in a thermostatted bath at $298.15 \mathrm{~K}$ and the calculated accuracy of temperature was lower than $0.1 \mathrm{~K}$ ). The mixtures of cationic/anionic surfactants easily formed precipitates in aqueous solution due to their strong interactions. Hence, the experiments were conducted in the anionic-rich region. Ferrocene (Sigma-Aldrich, 98\% pure) and (2,2,6,6-tetramethylpiperidin-1-yl) oxidanyl (TEMPO, Merck, 99\% pure) were also used without recrystallization.

\subsection{Methods}

\subsubsection{Surface Tension Measurements}

Surface tension measurements were conducted using a tensiometer (Krüss K12) by the ring method under atmospheric pressure [14]. Before each measurement a clean, flame-dried, platinum ring was vertically dipped and pulled into/out of the liquid and the maximum required force to pull the ring from the interface was calculated as the surface tension, $\gamma\left(\mathrm{mN} \cdot \mathrm{m}^{-1}\right)$. The tensiometer was calibrated at $298.15 \mathrm{~K}$ with pure water and the cleanliness of the glassware had been checked. In all cases, more than three successive 
measurements were carried out, and the standard deviation did not exceed $\pm 0.1 \mathrm{mN} \cdot \mathrm{m}^{-1}$. The temperature was controlled within $\pm 0.1 \mathrm{~K}$ by circulating thermostated water through the jacketed glass cell. The sample solution was continuously stirred using a magnetic stirrer. The uncertainty of the measurements is $\pm 0.1 \mathrm{mN} \cdot \mathrm{m}^{-1}$.

\subsubsection{Electrical Conductivity Measurements}

Conductivity measurements were carried out at 298.15 K with a Metrohm conductometer (model 771). The conductivity cell (Tacussel, France, XE 100) was made of two square platinum electrodes embedded in glass. The cell was immersed in the surfactant solution that was kept in a double-walled temperature-controlled glass container. After any injection, the solution was stirred and allowed to equilibrate for $10 \mathrm{~min}$, and then three successive measurements of specific conductivity were performed. The uncertainty of the measurements is $\pm 0.01 \mu \mathrm{S} \cdot \mathrm{cm}^{-1}$.

\subsubsection{Voltammetry Measurements}

Electrochemical measurements were performed at $298.15 \mathrm{~K}$ using a Biologic potentiostat (VSP300), with electro-active probes of (2,2,6,6-tetramethylpiperidin-1-yl) oxidanyl (TEMPO) and ferrocene, a three-electrode system composed of an $\mathrm{Ag} / \mathrm{AgCl}$ reference electrode, a platinum disk electrode $\left(0.0314 \mathrm{~cm}^{2}\right)$ as the working electrode, and a platinum foil $\left(2 \mathrm{~cm}^{2}\right)$ as the counter electrode, for CV measurements. All solutions were prepared to a final volume of $25 \mathrm{~mL}$, containing $0.1 \mathrm{~mol} \cdot \mathrm{L}^{-1} \mathrm{KCl}, 0.001 \mathrm{~mol} \cdot \mathrm{L}^{-1}$ ferrocene, $0.002 \mathrm{~mol} \cdot \mathrm{L}^{-1} \mathrm{TEMPO}$, and different concentrations of surfactants. Due to the insolubility of ferrocene in water, ferrocene was first dissolved in ethanol and then small volume of the resulting ethanol solution was added to the aqueous surfactant solution. The small volume of the ethanol solution to the final solution volume indicates that the effect of ethanol was negligible. After every measurement, surfaces of the working and counter electrodes were carefully cleaned by abrasion and washing with nitric acid. Electrodes were plunged in solutions for $10 \mathrm{~min}$, and then voltammograms were recorded for each solution with a scan rate of $100 \mathrm{mV} \cdot \mathrm{s}^{-1}$, and the fifth cycles were saved.

\subsubsection{TEM, DLS, and Zeta Potential Measurements}

The DLS and Zeta potential measurements were conducted with a Zetasizer Nano (Malvern, MRK825-02, UK). The DLS instrument was calibrated against NIST-traceable particle size standards (Polysciences, Inc. USA). Solutions were prepared and rendered to the operator. TEM images were recorded with a Philips CM $120(120 \mathrm{kV})$. Samples were drop-casted on carbon coated grids and after solvent vaporization the micrographs were captured.

\section{Results and Discussion}

\subsection{CMC and Interfacial Properties}

The surface tension and conductivity measurements as illustrated in Fig. 1a and b are typical plots for the SDS/CTAB mixture (in the anionic-rich regime) in aqueous mixtures 

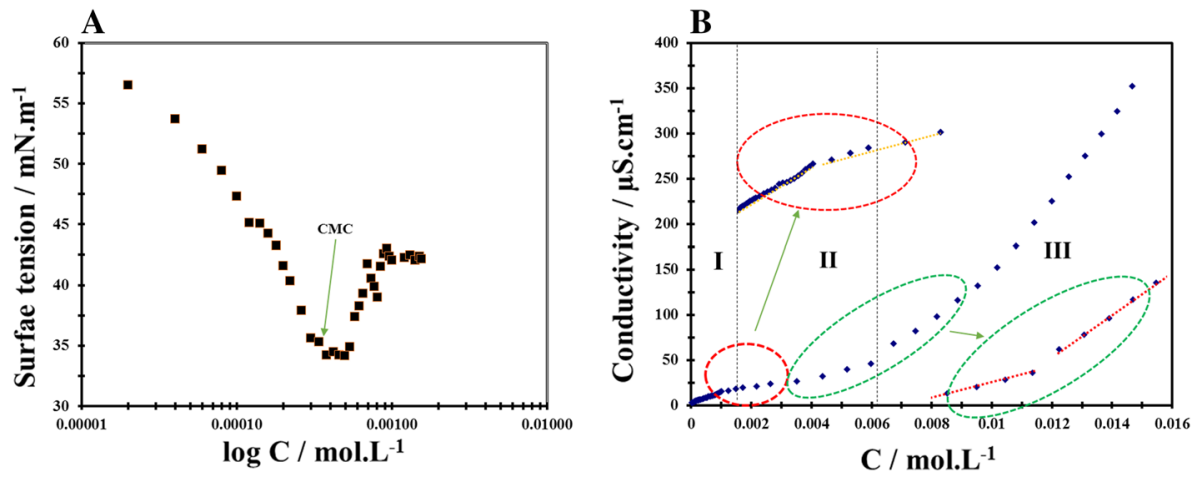

Fig. 1 a Plot of the surface tension and $\mathbf{b}$ plot of the specific conductivity versus the total concentration in solution of water and $\mathrm{EG}\left(X_{\mathrm{EG}}=0.1, \alpha_{\mathrm{SDS} / \mathrm{CTAB}}=98: 2\right)$ at $298.15 \pm 0.1 \mathrm{~K}$

of EG. At the point where micelles began to form, the surface tension (Fig. 1a) shows a clear break. That is, the concentration corresponding to the break in the curve indicates the CMC of each binary combination of the SDS/CTAB mixture. However, the conductivity behavior of the SDS/CTAB mixture in the anionic-rich regime (Fig. 1b) differed from that of the surfactants in the pure state or their mixture in the cationic regime [14]. The conductivity curve in Fig. $1 \mathrm{~b}$ included two break points in $C_{1}$ and $C_{2}$ (three distinct parts). In the SDS/CTAB mixed system, at a total concentration exceeding $C_{1}$ (regime II), the conductivity remained almost constant as the concentration increased. This plateau most likely corresponds to formation of large aggregates and micellar growth due to strong interactions between them. In regime III, the solution conductivity increased as the total concentration increased. Break point $C_{2}$ is referred to as the phase transition from large aggregates to normal micelles. The $C_{1}$ and $C_{2}$ values are given in Table 1 . However, the surface tension plot (see Fig. 1a) shows a dip at around the same value where there was a break in the conductance (see Fig. 1b). This break may be due to formation of a second state of aggregation, which arises because of a change in the micelle shape. This shape change was also evident in the data from DLS measurements (Table 3). For all the combinations (in the anionic-rich regime) of the binary SDS/CTAB mixtures a second CMC was obtained.

The CMC, $C_{1}$, and $C_{2}$ values for different combinations of the binary SDS/CTAB mixtures (in the anionic-rich regime) in aqueous mixtures of EG are shown in Table 1. The $\mathrm{CMC}$ or $C_{1}$ corresponds to the formation of large micellar aggregates because of strong synergism between them. Whereas, the second states of aggregation represented by $C_{2}$ is due to structural transformations at surfactant concentration well above the first CMC. Such micellar transitions for catanionic surfactant systems have been reported by other groups [14-17].

It can be seen in Fig. $1 \mathrm{~b}$ that the slopes of conductivity versus $C$ of the linear region above $C_{1}$ and $C_{2}$ are smaller than those below $C_{1}$. This is a consequence of counterion binding at the surface of the micellar aggregates. In other words, there is an effective loss of ionic charges since a number of counterion are confined to the micelle surface. The degree of counterion dissociation, $\alpha$, corresponds to the average number of counterions/ surfactant ions that dissociate from the micelle. This parameter can be estimated from the ratio between the slopes of the curves above and below the CMC (William's method) [18-20]. Values of $\alpha_{1}$ and $\alpha_{2}$ are calculated from the ratio of slopes of II/I and III/II, 


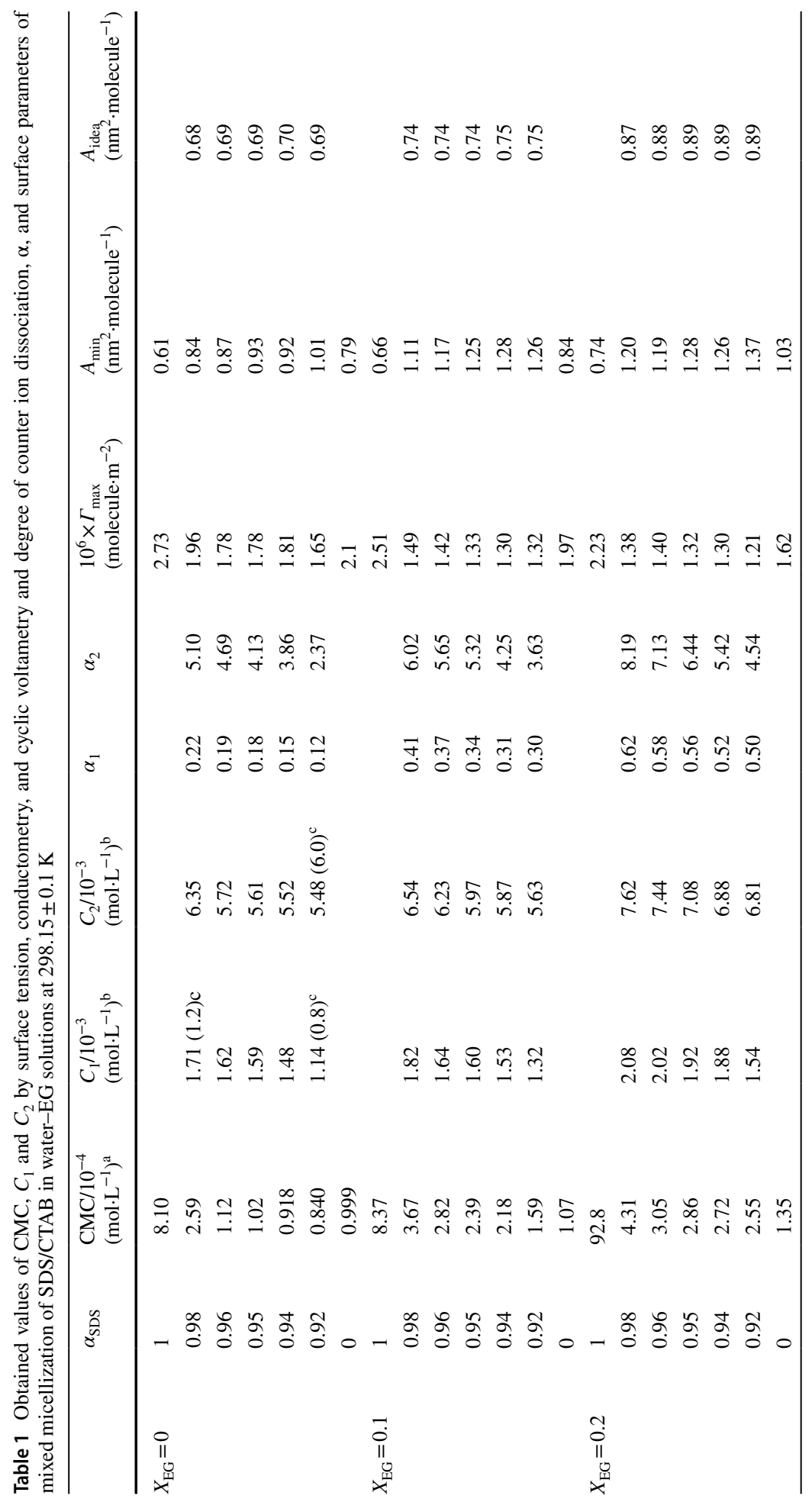




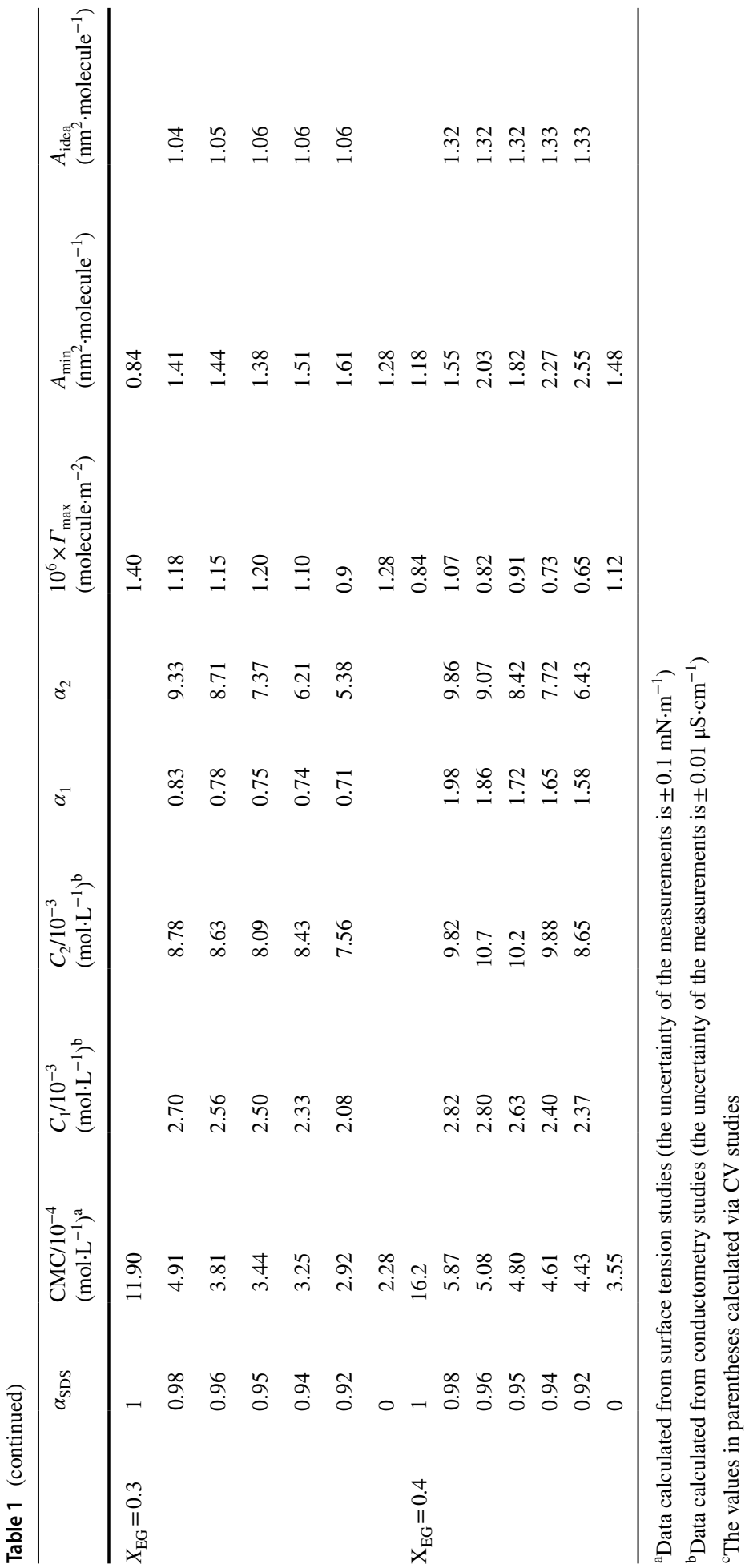


respectively. The values of $\alpha_{1}$ and $\alpha_{2}$ at various volume fractions of EG are listed in Table 1 for different SDS/CTAB anionic-rich mixtures. The increases observed in the $\alpha$ value as the EG volume fraction increased are probably due to the decreases in the charge density at the micellar surface, resulting from the decreases in the aggregation number in the aggregates. The phase transition from large aggregates to normal micelles is revealed by the values of $\Delta \alpha$. These results are in agreement with previous findings [6, 21].

Previously the effect of increasing the content of EG in the solution on the $\alpha$ and CMC was explained by studying the effects of dielectric constant in the medium [22]. Addition of EG affects both the interactions between hydrophobic groups and the interactions between hydrophilic groups in the surfactants. The dielectric constant of EG is much lower than that of water, so addition of EG to water will lower the dielectric constant of the mixed solvent. Therefore, addition of EG to aqueous solutions renders the hydrophobic effect of the catanionic surfactant molecules weaker. On the one hand, this is a disadvantage for micelle formation. On the other hand, addition of EG strengthens the attractive electrostatic interaction between their two oppositely charged polar groups, and consequently is an advantage for micelle formation. In SDS/CTAB mixtures, as a result, the competing effect of hydrophobic and electrostatic interactions on CMC offset each other due to EG addition, leading to only a small increase in the $\mathrm{CMC}$ values in the anionic-rich regime. The $\mathrm{CMC}$ values of SDS/CTAB mixtures decreased via addition of EG in the cationic-rich regime [14]. It can be seen that the presence of EG in the bulk phase affects the micellization process of the SDS/CTAB, pure CTAB and SDS systems. An increase in the volume fraction of EG causes the micellization process to occur less spontaneously. This can be understood on the basis of a reduction in the solvophobic interactions caused by improved solvation in the presence of EG, which leads to an increase in the solubility of the hydrocarbon tails and electrostatic repulsion between head groups (in ionic systems), which result in an increase in the CMC.

There are reports on the direct use of an electroactive probe's peak current $\left(i_{\mathrm{p}}\right)$ to determine the diffusion coefficients and, consequently, the sizes of the aggregates [14]. The variation in the $i_{\mathrm{p}}$ parameter after changing the concentration ratio of surfactant from 98:2 to 92:8 SDS/CTAB mixtures is presented in Fig. 2. Initially, addition of small quantities of surfactant mixture resulted in a small change in the $i_{\mathrm{p}}$ value, due to very weak interactions between the electroactive probe and the monomeric surfactant [23, 24]. These interactions are found to be a result of the weak hydrophobic environment available for solubilization of the probe. A further increase in the surfactant concentration leads to micelle formation, which facilitates the solubilization of the electroactive

Fig. 2 The effect from changing the total concentration for mixed solutions of SDS and CTAB in aqueous solutions $\left(X_{\mathrm{EG}}=0\right)$ on the peak current at $298.15 \pm 0.1 \mathrm{~K}$ : (Open square), 92:8 SDS/CTAB; (Filled square), 98:2 SDS/CTAB

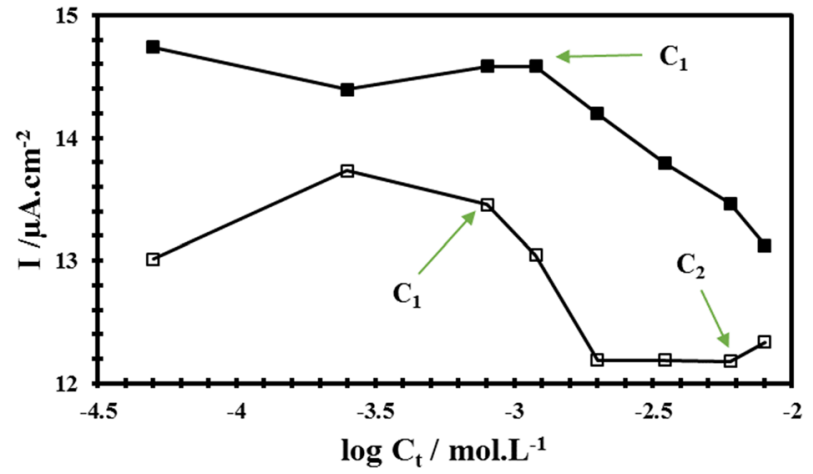


probe. The TEMPO in the micellar phase instantaneously reduces $i_{\mathrm{p}}$, which therefore tends to a negligible constant value. $C_{1}$ and $C_{2}$ values were obtained from a plot of $i_{\mathrm{p}}$ versus total surfactant concentration (Table 1 ). These values agreed with the conductometry results. In CV measurements, addition of supporting electrolyte does not significantly change the CMC values. These results are in agreement with previous findings [25]. For the 98:2 SDS/CTAB mixture, a discontinuity in the $i_{\mathrm{p}}$ versus concentration plot was observed. This discontinuity corresponded to the value of $C_{1}$ of this system. However, for the 92:8 SDS/CTAB mixtures, two discontinuities in the $i_{\mathrm{p}}$ versus concentration plot were observed. These discontinuities correspond to $C_{1}$ and $C_{2}$ of this system (in agreement with the results of the conductometry measurements). Cyclic voltammograms for the one-electron oxidation of TEMPO in SDS/CTAB for potential range of $0.0-0.8 \mathrm{~V}$ are given in Fig. SM1.

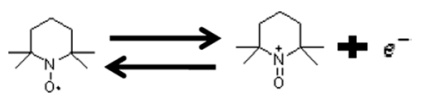

The $i_{\mathrm{p}}$ value (from CV studies) for an electroactive redox probe was calculated by the Randles-Sevcik equation [26].

$$
i_{p}=0.4463 F A C n\left(\frac{n F v D}{R T}\right)^{\frac{1}{2}}
$$

where $n$ is the number of electrons involved in oxidation or reduction, $A$ is the area of the electrode ( $A$ was determined by the method described in the supporting materials (SM1)), $F$ is Faraday's constant, $R$ is the gas constant, $T$ is the absolute temperature, $D$ is the diffusion coefficient of the TEMPO probe, $C$ is the TEMPO concentration in the solution, and $\nu$ is the scan rate. By assuming that the peak currents of the micellar probe and free probe are independent, while the probe is distributed between the micelles and the bulk phase, Eq. 3 was used as follows:

$$
D^{1 / 2}=\frac{i_{p}}{0.4463 F^{3 / 2} A C n^{3 / 2} R^{-1} T^{-1} \nu^{1 / 2}}=\frac{D_{m}^{1 / 2} K N_{a g g}^{-1}(C-C M C)+D_{f}^{1 / 2}}{1+K N_{a g g}^{-1}(C-C M C)}
$$

where $D$ is the observed diffusion coefficient in the micellar system, $D_{f}$ and $D_{m}$ are the TEMPO diffusion coefficients in the bulk and micelle phases, respectively. The micelle self-diffusion coefficient is monitored based on the cyclic voltammograms for the oneelectron oxidation of ferrocene, which can be assumed to be completely solubilized in the micellar phase. The solution of Eq. 3 yielded the partition coefficient $(K)$ of TEMPO between the bulk and micellar phases. It was found that the $K$ value is about $1.3 \times 10^{7}$ and $4.5 \times 10^{4} \mathrm{~L} \cdot \mathrm{mol}^{-1}$ for 98:2 and 92:8 SDS/CTAB, respectively, in the CMC region. No sharp variation in solubility may be explained by the size and shape of aggregates that are almost constant. The present results are consistent with findings of previous studies, that have shown the probe is sensitive to the surfactant concentration, and can mirror the changes taking place within the structure of the aggregates [27]. It is not possible to perform CV measurements for SDS/CTAB mixtures in water-EG solutions. This is due to an overlap in the cyclic voltammograms for EG and TEMPO.

The Gibbs equation at diluted solutions is considered as an effective method for determination of surface excess concentration, $\Gamma_{\max }$, at the air-liquid interface of surfactant adsorption [21]: 


$$
\Gamma_{\max }=\frac{-1}{2.303 n R T}\left[\frac{d \gamma}{d \log _{10} C}\right]_{T, P}
$$

Here, $R, T, C, \gamma$ and $n$ are gas constant, temperature $(\mathrm{K})$, the concentration of surfactant, the surface tension, and the number of formed species from dissociation per monomer, respectively. The minimum area per head group $\left(A_{\min }\right)$ for surfactant molecules at the CMC at the saturated interface was obtained by the following equation [21]:

$$
A_{\min }=\frac{10^{18}}{N_{A} \Gamma_{\max }}\left(\mathrm{nm}^{2} \cdot \text { molecule }^{-1}\right)
$$

The $\Gamma_{\max }$ value was calculated by a curve fitting of the plot of $\gamma$ versus $\log _{10} C$ and $N_{\mathrm{A}}$ is Avogadro's number. The curve fitting was conducted by means of a polynomial equation (PE: $\left.y=a x^{2}+b x+C\right)$. Then, the parameters of $\Gamma_{\max }($ at the CMC) were calculated by determining the slope of the tangent [27]. The fits have $\mathrm{R}^{2}$ (regression coefficient) values between 0.9 and 0.99. As the Table 1 shows upon increasing the EG concentration, the values of $\Gamma_{\max }$ for SDS/ CTAB mixtures change uniformly. Several factors can be listed for the observed decrease in $\Gamma_{\max }$ which can be attributed to (1) the presence of EG at the interface, (2) the interaction between EG and the surfactant, and (3) a change in the water structure due to addition of EG [28]. Likely, with increasing the amount of EG, $A_{\min }$ shows an inverse behavior with respect to $\Gamma_{\max }$ (Table 1). Moreover, the small value of $A_{\min }$ (calculated in pure surfactant solutions) indicates that the air-liquid interface was closely packed, and the molecules of surfactant at this interface are oriented perpendicularly to the interface (Fig. SM2). The explanation proposed for the high $A_{\min }$ values in the SDS/CTAB mixtures, is the presence of the anionic-cationic ion pairs of surfactant (or at least a portion of them) on the surface (Fig. SM2). The ideal mixing values, $A_{\text {ideal }}$, that are presented in Table 1 , result from a calculation from of the equation: $A_{\text {ideal }}=Z_{1} A_{1}+\left(1-Z_{1}\right) A_{2}$, where $Z_{1}$ is the mole fraction of component 1 in the mixed monolayer, and $A_{1}$ and $A_{2}$ are the minimum area per molecule of SDS and CTAB, respectively. In spite of the strong synergism between $A_{\text {min }}$ and $A_{\text {ideal }}$, the experimental values for $A_{\min }$, are larger than $A_{\text {ideal }}$. It is possible that there is some ion pair formation. van der Waals self-attraction between the hydrophobic portions of each SDS molecule, before mixing, can potentially be reduced upon mixing with CTAB.

\subsection{Interaction Between Surfactants in the Aggregated Phase and at the Liquid-Air Monolayer Interface}

Using a model (developed by Rubingh et al. [29-31]), as a regular solution for determination of $\beta$ parameters in binary systems, gives us an evaluation of the nature and strength of the interactions between two surfactants.

The following equations were used to calculate the $\beta^{\mathrm{M}}$ interaction parameter for mixed micelle in our aqueous medium [32]:

$$
\begin{gathered}
\frac{\left(X_{1}^{M}\right)^{2} \log _{10}\left(\alpha_{1} C_{12}^{M} / X_{1} C_{1}^{M}\right)}{\left(1-X_{1}^{M}\right)^{2} \log _{10}\left[\left(1-\alpha_{1}\right)\right] C_{12}^{M} /\left(1-X_{1}^{M}\right) C_{2}^{M}}=1 \\
\beta^{M}=\frac{\log _{10}\left[\alpha_{1} C_{12}^{M} / X_{1} C_{1}^{M}\right]}{\left(1-X_{1}^{M}\right)^{2}}
\end{gathered}
$$


where at the mole fraction $\alpha_{1}, X_{1}^{M}, C_{1}^{M}, C_{2}^{M}$, and $C_{12}^{M}$ are the total mole fraction of surfactant 1 in the mixed micelle and the CMCs for surfactant 1 , surfactant 2, and their mixture, respectively.

Using the following equations at the aqueous solution/air interface, the interaction parameter for mixed monolayer formation $\beta^{\sigma}$, is calculated [30-32]:

$$
\begin{gathered}
\frac{Z_{1}^{2} \log _{10}\left(\alpha_{1} C_{12} Z_{1} C_{1}^{0}\right)}{\left(1-Z_{1}\right)^{2} \log _{10}\left[\left(1-\alpha_{1}\right)\right] C_{12} /\left(1-Z_{1}\right) C_{2}^{0}}=1 \\
\beta^{\sigma}=\frac{\log _{10}\left(\alpha_{1} C_{12} / Z_{1} C_{1}^{0}\right)}{\left(1-Z_{1}\right)^{2}}
\end{gathered}
$$

Here, in a surfactant-only basis and in the total mixed monolayer, the mole fraction of surfactant 1 is represented by $Z_{1}$, and in the solution phases, the molar concentrations of surfactant 1 , surfactant 2 , and their mixture, are $C_{1}^{0}, C_{2}^{0}$, and $C_{12}$, respectively, required to produce a given $\gamma$ value (at mole fraction $Z_{1}$ of surfactant 1). In these experiments, the $C_{1}$, $C_{2}$, and $C_{12}$ values were determined corresponding to a surface tension of $\gamma=34.5 \mathrm{mN} \cdot \mathrm{m}^{-1}$. In order to evaluate $Z_{1}$, Eq. 8 was numerically solved and then substituted into Eq. 9 for the calculation of $\beta^{\sigma}$. The interaction parameter calculated through Eqs. 7 and 9, according to the Rubingh model (Table SM1), must be independent of composition of the micelles. But, through the application of this model, $\beta^{\mathrm{M}}$, a composition-dependent interaction parameter, is yielded. The calculated values for $\beta_{\mathrm{ave}}^{\mathrm{M}}$ are presented in Table 2. This $\left|\beta_{\mathrm{ave}}^{\mathrm{M}}\right|$ value, for different degrees of nonideality of this interaction in mixed micelles, can shed light on the strength of the degree of interaction between two surfactants. Strong attractive interaction between SDS and CTAB can be inferred from a large negative values of $\beta^{\mathrm{M}}$. As the results in Table 2 show, the $\left|\beta_{\text {ave }}^{\mathrm{M}}\right|$ slightly decrease after addition of EG, which can be attributed to the structure-breaking nature of EG in water and a reduction in the hydrophobic interactions, which are the main driving forces for mixed micellization. Moreover, at higher volume of $\mathrm{EG}$, the $\left|\beta_{\mathrm{ave}}^{\mathrm{M}}\right|$ value also increases, which can be attributed to the electrostatic attraction increase upon mixing between the two polar ionic head groups with opposite charges of SDS and CTAB. Interestingly, in cationic-rich regime mixtures of ionic/nonionic or SDS/CTAB, the $\left|\beta_{\text {ave }}^{\mathrm{M}}\right|$ values show the same trend in (after increasing EG content for SDS/CTAB) [33] and [14]. The behaviors of $\left|\beta_{\text {ave }}^{\mathrm{M}}\right|$ and $\left|\beta_{\text {ave }}^{\sigma}\right|$ after EG addition have similar patterns (Table 2). Here, the interaction parameter $\left|\beta_{\text {ave }}^{\sigma}\right|$, shows a minimum upon increasing the volume percentage of EG (from $0 \%$ to $40 \%$ ). As the EG is added to the solution $\left(X_{\mathrm{EG}}=0.1\right)$, the value of $\left|\beta_{\text {ave }}^{\sigma}\right|$ decreases. The initial decrease in $\left|\beta_{\mathrm{ave}}^{\sigma}\right|$ as $\mathrm{EG}\left(X_{\mathrm{EG}}=0.1\right)$ added to the aqueous solution is attributed to a reduction in the attractive van der Waals

Table 2 Cosolvent effect on $\beta_{\text {ave }}^{\mathrm{M}}$ and $\beta_{\text {ave }}^{\sigma}$ values

\begin{tabular}{llll}
\hline$X_{\mathrm{EG}}$ & $\beta_{\mathrm{ave}}^{\mathrm{M}}$ & $\beta_{\mathrm{ave}}^{\sigma}$ & $\beta_{\text {ave }}^{\sigma}-\beta_{\text {ave }}^{\mathrm{M}}$ \\
\hline 0 & $-16.56(-7.86)$ & $-18.31(-8.26)$ & $-1.75(-0.4)$ \\
0.1 & $-13.21(-6.92)$ & $-13.96(-5.80)$ & $-0.75(-1.12)$ \\
0.2 & $-13.37(-11.91)$ & $-14.17(-12.70)$ & $-0.80(-0.79)$ \\
0.3 & -14.88 & -16.10 & -1.22 \\
0.4 & -15.52 & -16.80 & -1.28 \\
\hline
\end{tabular}

The values in parentheses obtained from [14] 
interactions between hydrophobic groups at the planar air/aqueous solution interface, due to the structure-breaking nature of EG in water and the presence of EG in the monolayer at the air/aqueous solution interface. The increase in $\left|\beta_{\text {ave }}^{\sigma}\right|$ at higher volume percentages of $\mathrm{EG}\left(X_{\mathrm{EG}}=0.2-0.4\right)$ could result from increases in the electrostatic attraction between ionic hydrophilic groups through mixing. The variations in $\left|\beta_{\text {ave }}^{\sigma}\right|$ and $\left|\beta_{\text {ave }}^{\mathrm{M}}\right|$ following addition of EG in the cationic-rich mixtures [14] are larger than those variations in the anionic-rich mixtures. In CTAB, the charged trimethylammonium group provides a high charge density, which in turn gives rise to a high repulsive electrostatic interaction. In SDS, however, the charge is distributed over three oxygen atoms, leading to a lower surface charge density and consequently to a lower repulsive electrostatic interaction. As a result, an increase in EG content results in greater decreases in the electrostatic repulsion between the cationic groups in cationic-rich mixtures than those in anionic-rich mixtures. Furthermore, it is shown that the value of $\beta_{\mathrm{ave}}^{\sigma}-\beta_{\mathrm{ave}}^{\mathrm{M}}$ at first increases from increasing of the EG concentration $\left(X_{\mathrm{EG}}=0.1\right)$, reaches a maximum at a specific concentration $\left(\mathrm{X}_{\mathrm{EG}}=0.1\right)$, and thereafter decreases with further increases of the EG concentration $\left(X_{\mathrm{EG}}=0.2-0.4\right)$ (Table 2). This suggests that such electrostatic attraction increases among the polar head groups with opposite charges (SDS and CTAB). Such attraction, at the planar air and aqueous interface shows a greater effect than at the convex micellar surface in an aqueous medium, after increasing the concentration of EG (up to 10\%) in the solution. Overall, the results show that the energy of the electrostatic attractions generated at the planar interface is noticeably greater than that in the micelle. The results of this part are supported by previous findings in cationic rich-mixtures [14].

\subsection{Size, Morphology, Interaction of Catanionic Self-Assemblies and Zeta Potentials of the Aggregates}

$\mathrm{CV}$ measurements were used to determine the diffusion coefficients of the aggregates and the inter particle interaction parameters [33]. For this section, ferrocene was chosen as the electroactive probe; ferrocene can be used, provided that it does not perturb the micelle, and provided that its rates of entrance/exit into the aggregates with fast and reversible electron transfer are at least comparable to those of the surfactant monomers. The electrochemistry of ferrocene in nonaqueous, aqueous, and micellar environments has been described elsewhere [34-37].

$$
F c \rightleftharpoons F c^{+}+e^{-}
$$

In self-assembling systems with a fully solubilized electroactive probe that diffuses into the aggregates, the diffusion coefficient in aggregates is $D_{\mathrm{agg}}$. The ferrocene concentration was fixed at $1.0 \mathrm{mmol} \cdot \mathrm{L}^{-1}$. Hence, for various aggregate concentrations, based on the slope of the $i_{\mathrm{p}}$ versus $\nu^{1 / 2}$ plot, the $D_{\text {agg }}$ value could be obtained. The value of $D_{\text {agg }}$ after increasing the concentrations of SDS/CTAB mixtures (in EG solutions) was found to decrease (Fig. 3). Based on previous reports, the concentration of salt does not significantly affect the properties in a mixture of such surfactants [25]. At concentrations close to the CMC, the aggregates interact with each other, so the calculated values for the $D_{\text {agg }}$ are lower in comparison with the calculated $D_{\text {agg }}$ at the CMC. In order to calculate the inter-particle interaction parameters, the linear interaction theory was used [38, 39]. Consequently, extrapolation to infinite dilution (i.e., to the CMC) yields mass-transport parameters that are independent of inter particle interactions but are linked via Eq. 11 as: 

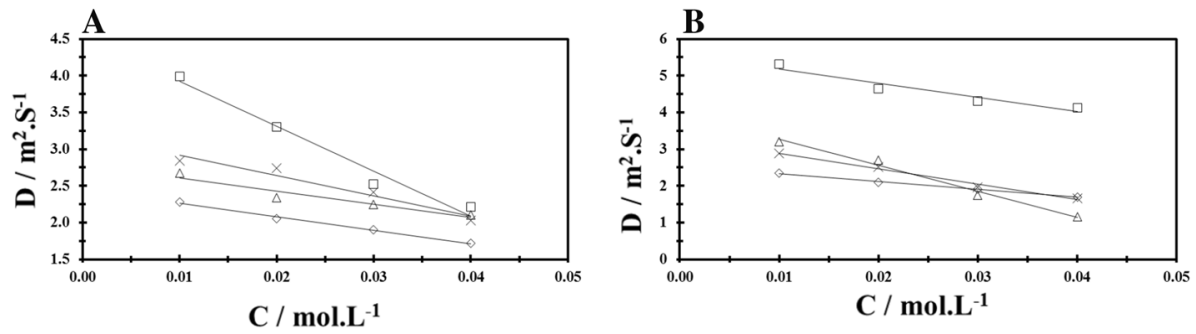

Fig. $3 D_{\text {agg }}$ versus the total concentration of surfactants for: a 98:2 SDS/CTAB and b 92:8 SDS/CTAB mixtures in: (Open diamond), $\mathrm{H}_{2} \mathrm{O}$; (Open square), $X_{\mathrm{EG}}=0.1$; (Open triangle), $X_{\mathrm{EG}}=0.2$; (Mutiple sign), and $X_{\mathrm{EG}}=0.3$, all at $298.15 \pm 0.1 \mathrm{~K}$

$$
D_{\mathrm{agg}}=D_{\mathrm{agg}}^{0}\left[1-k_{\mathrm{d}}\left(C_{\mathrm{t}}-\mathrm{CMC}\right)\right]
$$

Here, $C_{\mathrm{t}}, D_{\text {agg }}^{0}$ and $k_{\mathrm{d}}$ are the surfactant concentration, the self-diffusion coefficient without particle interaction and the inter particle interaction parameter, respectively. Figure 3 shows the dependence of $D_{\text {agg }}$ on aggregate concentration. It is possible that it is due to both attractive and repulsive interactions as well as micellar growth. In Eq. 11, the slope, intercept at $C_{\mathrm{t}}=\mathrm{CMC}_{2}$ and $k_{\mathrm{d}}$ are respectively equal to $-D_{\mathrm{agg}}^{0} k_{\mathrm{d}}, D_{\mathrm{agg}}^{0}$ and $k_{\mathrm{d}}=-$ slope/intercept (the $k_{\mathrm{d}}$ values have been calculated in concentration range of $C_{\mathrm{t}}>10 \mathrm{mmol} \cdot \mathrm{L}^{-1}$ so that no phase transition occurred). As the results in Table 3 show, changing in the mole fraction of cationic surfactant affects the $k_{\mathrm{d}}$ values. The attractive interactions between aggregates are increased by addition of a cationic surfactant, resulting in the slight decrease in $k_{\mathrm{d}}$ observed for these aggregate systems. The negligible variation of $k_{\mathrm{d}}$ from 8.1 to $7.6 \mathrm{~L} \cdot \mathrm{mol}^{-1}$ by increasing the CTAB mole fraction indicates that no change in morphology and size has occurred. These results were confirmed from DLS and $\xi$-potential results. Micelles with diameters of 3.5 and $4.5 \mathrm{~nm}$ at $16 \mathrm{mmol} \cdot \mathrm{L}^{-1}$ surfactant concentration are noticed for $98: 2$ and 92:8 SDS/CTAB mixtures in water (Table 3). The zeta potential remained constant upon increasing of the CTAB mole fraction (Table 3). In both mole fractions, at low concentrations $\left(C_{\mathrm{t}}<5 \mathrm{mmol} . \mathrm{L}^{-1}\right)$, large aggregates with diameter $\sim 5-100 \mathrm{~nm}$ were formed. An increase in the concentration resulted in a sudden decrease in the diameter: 3.5 and $4.5 \mathrm{~nm}$, respectively, for the two fractions $98: 2$ and 92:8. This indicates that the transition of the super-molecular structure to normal micelle is consistent with that of the conductivity zeta potential results. From the TEM image in the case of 92:8 composition, the presence of large aggregates at low concentration $\left(4 \mathrm{mmol} \cdot \mathrm{L}^{-1}\right)$ can be readily inferred (Figs. 4 and 5). The average diameter of the aggregates $(\sim 50 \mathrm{~nm}$ and $30 \mathrm{~nm})$ is consistent with the diameter determined from DLS measurements $\left(X_{\mathrm{EG}}=0\right.$ and 0.1$)$. It has already been shown in a previous work that the formation of spherical micelles (with diameter $\sim 50 \mathrm{~nm}$ ) occurs at a higher SDS concentration (SDS concentration $=10 \mathrm{mmol} \cdot \mathrm{L}^{-1}$ and CTAB concentration $\left.=0.1 \mathrm{mmol} \cdot \mathrm{L}^{-1}\right)\left(X_{\mathrm{EG}}=0\right)[40]$. As shown in Table 3, by increasing the EG content in the aqueous solution to $10 \%$, the size and surface potential of SDS/CTAB aggregates in the anionic region remained constant, and no dramatic structural change took place in the solutions in low concentrations $\left(4 \mathrm{mmol} \cdot \mathrm{L}^{-1}\right)$. As shown in Fig. 4, TEM images confirmed the DLS results. However, at high concentrations, in the presence of $10 \%$ EG, the SDS/ CTAB systems (except the 98:2 SDS/CTAB system) did not display behavior similar to that shown by SDS/CTAB in pure water. According to the data presented in Table 3 , the $k_{\mathrm{d}}$ values for the SDS/CTAB systems increased with decreasing anionic surfactant content in 


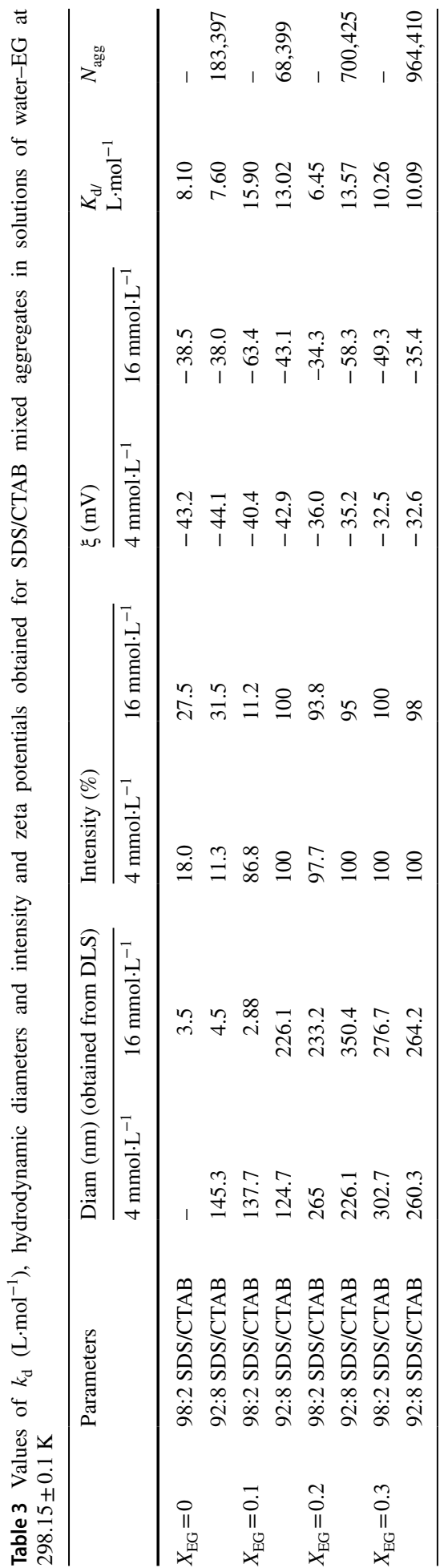




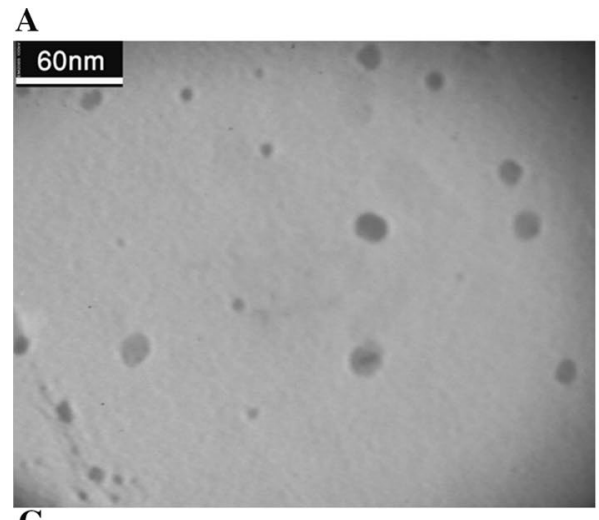

B
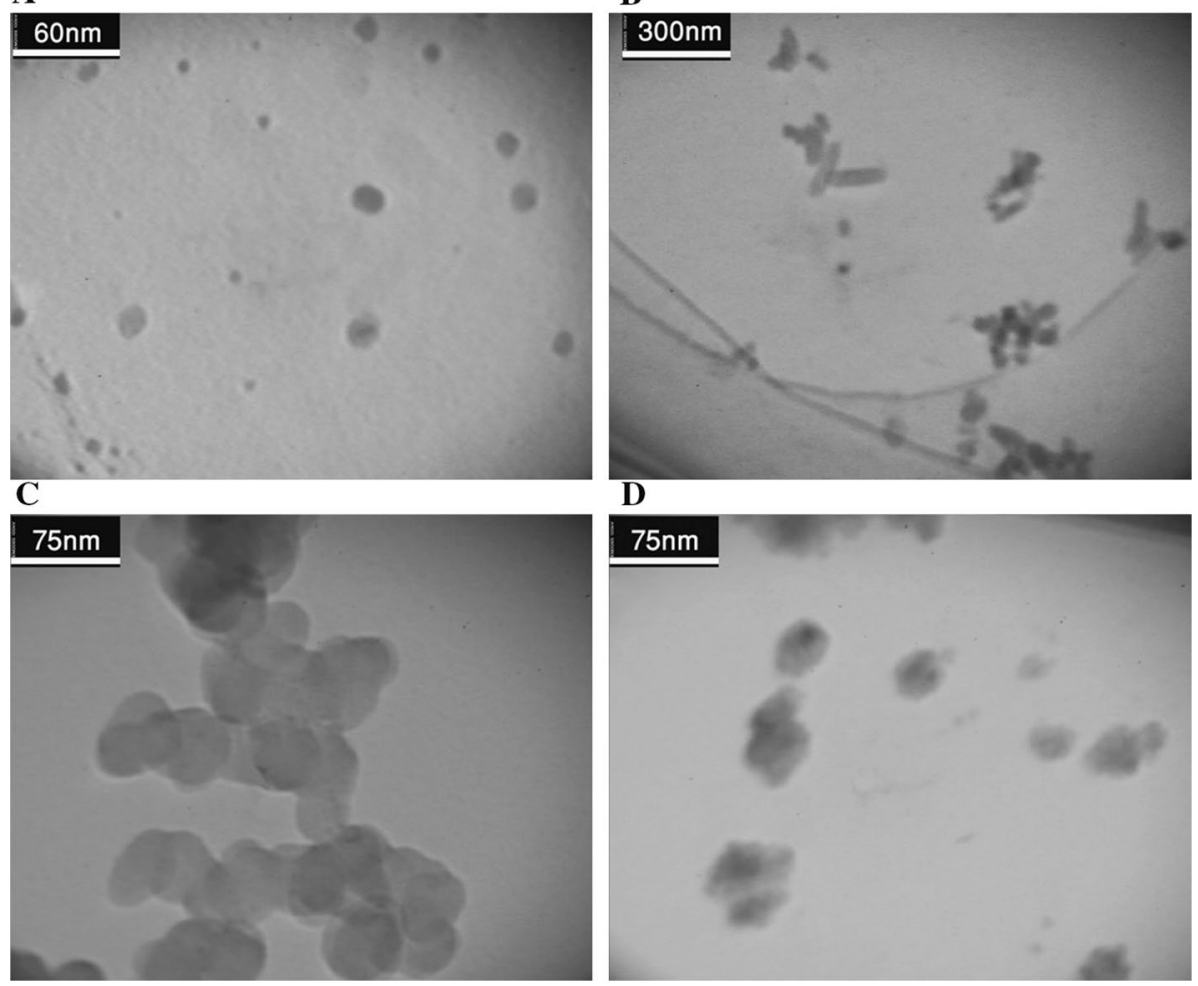

Fig. 4 The TEM images of mixtures of SDS/CTAB at: a 92:8 (after 12 h, $X_{\mathrm{EG}}=0$ ), b 92:8 (after $12 \mathrm{~h}$, $X_{\mathrm{EG}}=0.1$ ), c 92:8 (after $12 \mathrm{~h}, X_{\mathrm{EG}}=0.2$ ), and d 92:8 (after $12 \mathrm{~h}, X_{\mathrm{EG}}=0.3$ )

the presence of $20 \%$ EG. In spite of the decrease in micellar surface charge density from the decrease of SDS (Table 3), an increase in $k_{\mathrm{d}}$ values was observed. This unexpected increase in $k_{\mathrm{d}}$ values can be mainly attributed to the cylindrical-to-spherical morphology change. Representative DLS results of 92:8 SDS/CTAB mixed micelles at 20\% EG confirmed the above results (Table 3). By further addition of EG, the $k_{\mathrm{d}}$ values increased to approximately $10 \mathrm{~L} \cdot \mathrm{mol}^{-1}$ in $30 \%$ EG solution and were unchanged upon decreasing the SDS content. The lack of variation of $k_{\mathrm{d}}$ observed indicates that the morphology does not change significantly from decreasing the SDS mole fraction. Moreover, the aggregation of spherical structures can be seen for $X_{\mathrm{EG}}=0.2$ and $X_{\mathrm{EG}}=0.3$ (Fig. 4).

\section{Conclusion}

The self-aggregation behavior, in a water/EG mixed solvents, of binary composition of SDS/CTAB with an excess of anionic surfactant, was examined by surface tension, conductivity, DLS, TEM, and CV measurements. Depending on the EG content of the solvent, the SDS/CTAB self-assembled into configurations ranging from cylindrical to spherical. To evaluate the effects of cosolvents on the surfactant-surfactant interaction in binary anionic/cationic surfactants, the values of $\beta$ parameters were calculated. It was shown that 

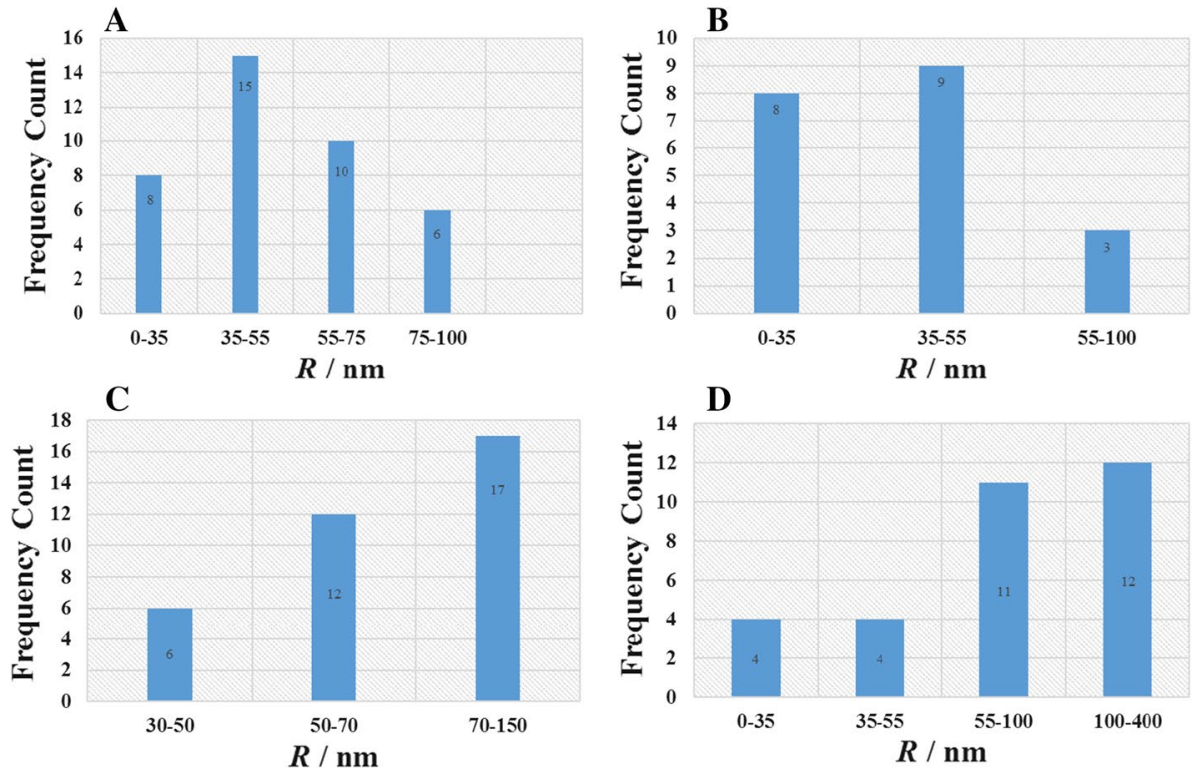

Fig. 5 Size distribution plots of mixed aggregates: a $X_{\mathrm{EG}}=0,92: 8$ SDS/CTAB; $\mathbf{b} X_{\mathrm{EG}}=0.1$, 92:8 SDS/ $\mathrm{CTAB} ; \mathbf{c} X_{\mathrm{EG}}=0.2,92: 8 \mathrm{SDS} / \mathrm{CTAB} ; \mathbf{d} X_{\mathrm{EG}}=0.3,92: 8 \mathrm{SDS} / \mathrm{CTAB}$. The total surfactant concentration was fixed at $4 \mathrm{mmol} \cdot \mathrm{L}^{-1}$ )

adding EG to the solution reduces the magnitude of the interaction parameters in both mixed micelle $\left(\beta_{\mathrm{ave}}^{\mathrm{M}}\right)$ and mixed monolayer $\left(\beta_{\mathrm{ave}}^{\sigma}\right)$. This indicates a greater effect on the interaction in the mixed monolayer in comparison with the mixed micelle. At a higher volume fraction of EG, the synergism effect of mixed aggregates is significantly increased, which is attributed to the increase in attractive electrostatic interaction between oppositely charged head groups upon mixing.

Open Access This article is distributed under the terms of the Creative Commons Attribution 4.0 International License (http://creativecommons.org/licenses/by/4.0/), which permits unrestricted use, distribution, and reproduction in any medium, provided you give appropriate credit to the original author(s) and the source, provide a link to the Creative Commons license, and indicate if changes were made.

\section{References}

1. Eivazihollagh, A., Svanedal, I., Edlund, H., Norgren, M.: On chelating surfactants: molecular perspectives and application prospects. J. Mol. Liq. 278, 688-705 (2019)

2. Abe, M., Scamehorn, J.F.: Surfactant Science Series; Mixed Surfactant Systems. CRC Press, Boca Raton (2004)

3. Scamehorn, J.F.: Phenomena in Mixed Surfactant Systems. ACS Symposium Series 311. ACS, Washington, DC (1986)

4. Somasundaran, P.: Encyclopedia of Surface and Colloid Science. Taylor \& Francis, Boca Raton (2015)

5. Shiloach, A., Blankschtein, D.: Predicting micellar solution properties of binary surfactant mixtures. Langmuir 14, 1618-1636 (1998)

6. Zhu, B.Y., Rosen, M.J.: Synergism in binary mixtures of surfactants: IV. Effectiveness of surface tension reduction. J. Colloid Interface Sci. 99, 435-442 (1984) 
7. Holland, P.M., Rubingh, D.N.: Mixed Surfactant Systems. ACS Symposium Series, Chap. 1. ACS, Washington, DC (1992)

8. Torchilin, V.: Multifunctional Pharmaceutical Nanocarriers. Fundemental Biomedical Technologies. Springer, New York (2008)

9. Meyers, D.: Surfactants Science and Technology. VCH Publishers, New York (1988)

10. Palepu, R., Gharibi, H., Bloor, D.M., Wyn-Jones, E.: Electrochemical studies associated with the micellization of cationic surfactants in aqueous mixtures of ethylene glycol and glycerol. Langmuir $\mathbf{9}$, 110-112 (1993)

11. Treitel, O.: The thermoelastic behavior of cylindrical plant tissues. J. Colloid Interface Sci. 2, 453-462 (1947)

12. Liu, S., Mao, C., Niu, Y., Yi, F., Hou, J., Lu, S., Jiang, J., Xu, M., Li, C.: Facile synthesis of novel networked ultralong cobalt sulfide nanotubes and its application in supercapacitors. ACS Appl. Mater. Interfaces. 7, 25568-25573 (2015)

13. Evans, D.F., Yamauchi, A., Roman, R., Casassa, E.Z.: Micelle formation in ethylammonium, a low melting fused salt. J. Colloid Interface Sci. 88, 89-96 (1982)

14. Yousefi, A., Javadian, S., Gharibi, H., Kakemam, J., Rashidi-Alavijeh, M.: Cosolvent effects on the spontaneous formation of nanorod vesicles in catanionic mixtures in the rich cationic region. J. Phys. Chem. B 115, 8112-8121 (2011)

15. Sohrabi, B., Gharibi, H., Javadian, S., Hashemianzadeh, M.: A new model to study the phase transition from microstructures to nanostructures in ionic/ionic surfactants mixture. J. Phys. Chem. B 111, 10069-10078 (2007)

16. Yatcilla, M.T., Herrington, K.L., Brasher, L.L., Kaler, E.W.: Phase behavior of aqueous mixtures of cetyltrimethylammonium bromide (CTAB) and sodium octyl sulfate (SOS). J. Phys. Chem. 100, 58745879 (1996)

17. Rajkhowa, S., Mahiuddin, S., Dey, J., Kumar, S., Aswal, V.K., Biswas, R., Kohlbrecher, J., Ismail, K.: The effect of temperature, composition and alcohols on the microstructures of catanionic mixtures of sodium dodecylsulfate and cetyltrimethylammonium rromide in water. Soft Matter 13, 3556-3567 (2017)

18. Lianos, P., Lang, J.: Static and dynamic properties of dodium $p$-(1-propylnonyl) benzenesulfonate micelles. J. Colloid Interface Sci. 96, 222-228 (1983)

19. Dong, B., Li, N., Zheng, L., Yu, L., Inoue, T.: Surface adsorption and micelle formation of surface active ionic liquids in aqueous solution. Langmuir 23, 4178-4182 (2007)

20. Williams, R.J., Phillips, J.N., Mysels, K.J.: The critical micelle concentration of sodium lauryl sulphate at $25^{\circ} \mathrm{C}$. Trans. Faraday Soc. 51, 728-737 (1955)

21. Rosen, M.J.: Surfactants and Interfacial Phenomena. Wiley, Hoboken (2004)

22. Javadian, S., Kakemam, J.: Intermicellar interaction in surfactant solutions. A review study. J. Mol. Liq. 242, 115-128 (2017)

23. Aslanzadeh, S., Yousefi, A.: The effect of ethanol on nanostructures of mixed cationic and anionic surfactants. J. Surfactants Deterg. 17, 709-716 (2014)

24. Mahajan, R.K., Vohra, K.K., Shaheen, A., Aswal, V.K.: Investigations on mixed micelles of binary mixtures of zwitterionic surfactants and triblock polymers: a cyclic voltammetric study. J. Colloid Interfaces Sci. 326, 89-95 (2008)

25. Harada, M., Inada, Y., Nomura, M.: In situ time-resolved XAFS analysis of silver particle formation by photoreduction in polymer solutions. J. Colloid Interface Sci. 337, 427-438 (2009)

26. Sohrabi, B., Eivazzadeh, S., Sharifi, A., Azadbakht, R.: Self-assembled catanionic surfactant mixtures in aqueous/ionic liquid systems. J. Mol. Liq. 211, 754-760 (2015)

27. Mandal, A.B., Nair, B.U., Ramaswamy, D.: Determination of the critical micelle concentration of surfactants and the partition coefficient of an electrochemical probe by using cyclic voltammetry. Langmuir 4, 736-739 (1988)

28. Ruiz, C.C., Molina-Bolívar, J.A., Aguiar, J., MacIsaac, G., Moroze, S., Palepu, R.: Thermodynamic and structural studies of Triton X-100 micelles in ethylene glycol-water mixed solvents. Langmuir 17, 6831-6840 (2001)

29. Liley, J.R., Thomas, R.K., Penfold, J., Tucker, I.M., Petkov, J.T., Stevenson, P., Webster, J.R.P.: Surface adsorption in ternary surfactant mixtures above the critical micelle concentration: effects of asymmetry on the composition dependence of the excess free energy. J. Phys. Chem. B 121, 2825-2838 (2017)

30. Holland, P.M., Rubingh, D.N.: Review of mixed surfactant systems. J. Colloid Interface Sci. 158, 258 (1993). this article is a book review

31. Schulz, P.C., Rodríguez, J.L., Minardi, R.M., Sierra, M.B., Morini, M.A.: Are the mixtures of homologous surfactants ideal? J. Colloid Interface Sci. 303, 264-271 (2006) 
32. Rodríguez, A., Graciani, M.D.M., Cordobes, F., Moya, M.L.: Water-ethylene glycol cationic dimeric micellar solutions: aggregation, micellar growth, and characteristics as reaction media. J. Phys. Chem. B 113, 7767-7779 (2009)

33. Aghdastinat, H., Javadian, S., Tehrani-Bagha, A., Gharibi, H.: Spontaneous formation of nanocubic particles and spherical vesicles in catanionic mixtures of ester-containing gemini surfactants and sodium dodecyl sulfate in the presence of electrolyte. J. Phys. Chem. B 118, 3063-3073 (2014)

34. Ferreira, T.L., Sato, B.M., Seoud, O.A.E., Bertotti, M.: Application of microelectrode voltammetry to study the properties of surfactant solutions: alkyltrimethylammonium bromides. J. Phys. Chem. B 114, $857-862(2010)$

35. Moradi, P., Sohrabi, B., Najafi, M., Khani, V.: Adsorption and micellar phase properties of anionic surfactant in the presence of electrolyte and oil at different temperatures. Fluid Phase Equilib. 337, 370-378 (2013)

36. Zheng, P., Yin, H., Zhao, J., Shen, W.: Measurement of the interaction enthalpy for mixed micelles of dodecyltrimethylammonium bromide and 1-dodecyl-3-methylimidazolium bromide. J. Ind. Eng. Chem. 44, 204-209 (2016)

37. Zhang, L., Kang, W., Xu, D., Feng, H., Zhang, P., Li, Z., Lu, Y., Wu, H.: The rheological characteristics for the mixtures of cationic surfactant and anionic-nonionic surfactants: the role of ethylene oxide moieties. RSC Adv. 7, 13032-13040 (2017)

38. Dickinson, E.: Dispersions of interacting colloidal particles. Phys. Chem. 80, 3-37 (1983)

39. Santos, D.P., Longo, R.L.: Molecular dynamics simulations of specific anion adsorption on sulfobetaine (SB3-14) micelles. J. Phys. Chem. B 120, 2771-2780 (2016)

40. Xu, L., Zhao, W., Hao, J., Zhao, Y., Wang, D., Xu, H., Lu, J.R.: Self-assembly of magnetic bacillusshaped bilayer vesicles in catanionic surfactant solutions. Langmuir 32, 10226-10234 (2016)

Publisher's Note Springer Nature remains neutral with regard to jurisdictional claims in published maps and institutional affiliations. 